Vida Jesenšek*

Universität Maribor
UDK 811.112.2(497.412)(091)

DOI: 10.4312/linguistica.60.2.147-160

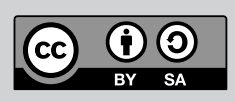

\title{
DEUTSCH IM HISTORISCHEN MARIBOR
}

\section{EINLEITUNG}

»Das Leben in Marburg ist ein durchaus deutsches, wenn auch gleich im nächsten Landleben schon slavisch modificirt; beider Sprachen sind fast die meisten heimischen Marburger mächtig, die dienende Classe gewöhnlich schon nach Einem in der Stadt zugebrachten Jahre ...«, so äußerte sich zum sprachlichen Bild der Stadt Mitte des 19. Jahrhunderts Rudolf Gustav Puff, Professor und Historiker deutscher (österreichischer) Herkunft an der damals bedeutendsten Bildungsinstitution in der Stadt, am deutschsprachigen Gymnasium (Puff 1847: 259). ${ }^{1}$ Dieses slavische Volk »hat seine Tugenden und Fehler; unter den Letzteren leider hervorstechend der Hang zur Unwahrheit und zum Trunke; unter Ersteren schnelles Auffassen, leichtes Sichschicken in alle Geschäfte« (ebd.) - wohl war mit dem Letzteren auch schnelles Erlernen der deutschen Sprache gemeint. Im Hinblick auf historische und wirtschaftliche Umstände, welche die Wahl der Umgangssprache ausschlaggebend im Zusammenhang mit dem sozialen Status der Sprecher regelten, ist die Aussage Puffs über die mehrheitliche Zweisprachigkeit in der Stadt mit Vorbehalt zu erachten, vor allem aber nicht im Sinne der heutigen Deutung des Zweisprachigkeitsbegriffs zu verstehen. Mit Zweisprachigkeit im historischen Maribor ist nicht die Kompetenz des Sprechers gemeint, sich in zwei Sprachen samt Vielfältigkeit ihrer Funktionen gleichwertig zu identifizieren, sich zwei Sprachgemeinschaften angehörig zu fühlen, zumal die Regelung für den Gebrauch beider Sprachen durch jeweilige historisch gegebene wirtschaftliche, politische und soziale Verhältnisse immer wieder neu festgelegt wurde. Beide Sprachen dienten, jeweils zu ihrer Zeit, zur Identifizierung der Bewohner mit ihrer Sprach- und Nationalgemeinschaft und zur Regelung der sozialen bzw. Machverhältnisse in Wirtschaft, Verwaltung, Alltagskommunikation, Schulwesen und Kulturleben. Beide Sprachen wurden, jeweils zu ihrer Zeit, als erstklassige Mittel politisch-kultureller Kämpfe ausgenutzt. Insbesondere heftig und aufrührerisch waren Ereignisse in Maribor (und gleichermaßen im gesamten Gebiet der altösterreichischen Untersteiermark) im Jahrhundert der politischen und kulturellen Umbrüche, d. h. von der Mitte des 19. bis zur Mitte des 20.

vida.jesensek@um.si

1 Der langdauernde deutsche Charakter der Stadt ist auch an der historischen Entwicklung des Stadtnamens nachvollziehbar. Die erste Erwähnung der slowenischen Stadtbenennung Maribor, geschaffen vom panslawistisch inspirierten Dichter Stanko Vraz, verzeichnet man erst in 1836, während vorher die Stadt mehrere Jahrhunderte unter varianten deutschsprachigen Namen bekannt war (Marchpurch, Marchburg, Marpurk, Marprog, Marburg an der Drau). Der slowenische Stadtname hat sich erst nach 1918 durchgesetzt. 
Jahrhunderts. Diese Epoche wird uns im weiteren Verlauf des Beitrags ${ }^{2}$ auch besonders interessieren. ${ }^{3}$

\section{ZWISCHEN DEUTSCH UND SLOWENISCH}

Der jahrhundertelange Kontakt zwischen der slowenischen und deutschen Sprache und Kultur hat die Stadt Maribor bedeutsam geprägt. Seit den Anfängen der Stadtentwicklung im 12. Jahrhundert (die Siedlung Marchburg war Teil der Markgrafschaft Ostfrankenreichs), vor allem aber seit dem 13. Jahrhundert, als die Stadt unter die Herrschaft der Habsburger kam, nahm die Zuwanderung aus den deutschsprachigen Ländern stark zu, vorrangig aus dem heutigen österreichischen Kärnten und der österreichischen Steiermark, jedoch auch aus Bayern und vielen deutschen mittelalterlichen Städten.

Ständische Berufsbezeichnungen ursprünglicher Stadtbewohner waren in der Regel deutsch: nadler, swertseger, puchsenmaister, glaser, arglmaister, pildmacher, pader ${ }^{4}{ }^{4}$ später lockte die Stadt auch den deutschen Adel an (vgl. z. B. den Mariborer Zweig des Geschlechts der Spannheimer (Bernhard von Spannheim)). Dank der schnellen Handwerksentwicklung und Handelsausbreitung zeichnete sich die Stadt bereits im 17. Jahrhundert durch einen multikulturellen und multiethnischen Charakter aus. So gab es in der Stadt zwischen der zweiten Hälfte des 18. und der ersten Hälfte des 19. Jahrhunderts 2,4-mal mehr fremde als einheimische Einwohner sowie 2,4-mal mehr Zuwanderer aus den deutschsprachigen als aus den slawischsprachigen Ländern (vgl. Curk 2002: 77). Die Zuwanderer (Handwerker, Handelsleute, Geistliche, Lehrer, Beamte, Künstler, später auch Industrielle) waren mehrheitlich deutschsprachig, in der Regel hochqualifiziert und gebildet; sehr schnell übernahmen sie führende Positionen in der Wirtschaft und Stadtverwaltung, dem gesellschaftlich-kulturellen Leben und Schulwesen. Vertreter niedrigerer sozialer Schichten der Stadtbevölkerung waren überwiegend slowenischsprachige Einheimische, oft Zuwanderer aus der ländlichen Umgebung. So konnten beide Sprachen sozial und funktional nicht gleichwertig verwendet werden, »Deutsch als Verständigungssprache war bis zur zweiten Hälfte des 19. Jahrhunderts in allen untersteiermärkischen Städten etwas Alltägliches« (Ferlež 2002: 93, übersetzt von V. J.). Dazu kam der Sprache

2 Beim vorliegenden Beitrag handelt es sich um eine leicht veränderte und übersetzte Version des slowenischen Artikels mit dem Titel Nemščina v Mariboru: preplet kulturnozgodovinskih in jezikovnih danosti. Vgl. Jesenšek (2019). Der Beitrag entstand im Rahmen der von der slowenischen Forschungsagentur (ARRS) finanzierten Untersuchungen zur slowenischen Identität und Kultur in Sprachkontakträumen (P6-0372).

3 Die Fachliteratur zum Thema ist relativ spärlich, dazu nicht selten ideologisch geprägt. Eine übersichtliche und dokumentarisch untermauerte Darstellung der deutschen Geschichte der Stadt Maribor leistete die Ausstellung Deutsche und Maribor: ein Jahrhundert der Wenden: 1846-1946, die im Frühling 2012 in Velika kavarna in Maribor lief. Vgl. den Ausstellungskatalog (Ferlež 2012).

4 Vgl. Bunc (1963; 175; übersetzt von V. J.): »Viele ständische Namen kennen wir in zwei Formen, in einer slowenischsprachigen und einer deutschsprachigen /.../ Dies geht darauf zurück, dass fremdsprachige Herrschafter unsere slowenischen Namen in ihren Urbaren und Standesbüchern gerne in der deutschen Übersetzung evidentiert haben. / . . / Angesichts dieser Praxis sollen wir nicht denken, dass alle unseren Šmids, Vebers, Fišers und Hafners in der Tat auch deutscher Herkunft sind.« 
zu jener Zeit noch keine nationalitäts- oder identitätsstiftende Rolle zu, »ein Bewohner von Maribor/Marburg, Celje/Cilli oder Ptuj/Pettau war in erster Linie ein Steirer, und dann ein bewusster Bürger und Lokalpatriot, der sich dem Deutsch- oder Slowenentum gegenüber neutral verhielt« (Ferlež 2012: 30). Der neuen politischen Nationalfrage war sich die regionale Bevölkerung noch nicht bewusst. Unter solchen wirtschaftlichen, politischen und sprachlichen Umständen erwischte die Stadt die zweite Hälfte des 19. Jahrhunderts, die Zeit des aufsteigenden National- und Sprachbewusstseins sowie potenzierter nationalpolitischen Spannungen in der Österreichischen Monarchie. Laut Cvirn (2016: 8) wollten die Untersteirer den gespannten Verhältnissen, Unstimmigkeiten und Feindseligkeiten ausweichen; in Celje und Maribor wurde sogar der Verein zur Vermittlung der slawischen und deutschen Nationalität gegründet, um, allerdings erfolglos, nationale Spannungen zu neutralisieren. Der alltägliche Sprachgebrauch hat sich zunächst im Wesentlichen noch nicht geändert: In der Alltagskommunikation und Verwaltung, im Handel, Handwerk und gesellschaftlichen Leben war Deutsch nach wie vor die Umgangs- und Amtssprache, wogegen Slowenisch in der Regel zur Verständigung mit sozial niedrigeren Schichten diente, man hat es »in Gesprächen „mit Ungebildeten“, Bauern, Schülern, Verwandten auf dem Dorf oder auch „nur zum Spaß“ gebraucht« (Ferlež 2012: 34).

$\mathrm{Zu}$ heftigeren nationalpolitischen und kulturellen Auseinandersetzungen zwischen den Deutschsprachigen und den Slowenen kam es in den 60er Jahren des 19. Jahrhunderts, in der Zeit nach der Einführung des Dualismus in der Monarchie. Zwischen 1868 und 1871 kam es in den slowenischsprachigen Gebieten zu großen Volksversammlungen, zu den sog. Tabori, um das slowenische Nationalbewusstsein, die Einführung des Slowenischen in die Bildung und Verwaltung sowie das politische Programm Vereinigtes Slowenien (Zedinjena Slovenija) ${ }^{5}$ zu fördern. Die Mariborer Deutschen haben diese nationalen Ideen erwartungsgemäß nicht unterstützt, mehr noch, sie antworteten mit einer Gegenbewegung, indem sie 1869 in mehreren untersteirischen Städten (Celje, Slovenska Bistrica, Radvanje) durch eigene Volksversammlungen für den deutschen Nationalstaat agierten.

Zur Bewahrung des Deutschtums in der Stadt haben wesentlich beigetragen auch die Methodologie und die Statistik der Volkszählungen zwischen 1880 und 1910. Jeweils ermittelt wurde unter anderem die Umgangssprache, wonach die Stadtbewohner entweder als Deutsche oder Slowenen geführt wurden. Angesichts der Tatsache, dass die deutsche Bevölkerung in der Wirtschaft, Verwaltung und im öffentlichen Leben überwog, war der Anteil der deutschen Umgangssprache sehr hoch und betrug mehr als $80 \%$. Diese teils manipulative Methodologie führte zu einer statistischen Assimilierung der Stadtbevölkerung. Die Stadtverwaltung war um die Jahrhundertwende gänzlich deutsch und vertrat die Auffassung, Maribor sei eine deutsche Stadt inmitten einer slowenischen Umgebung. Sie widersprach dem Mittelschulwesen mit slowenischer Unterrichtssprache, sie wollte sogar die Enthüllung des Denkmals für den Bischof Anton Martin Slomšek ${ }^{6}$ verhindern,

5 Das politische Programm Vereinigtes Slowenien aus dem Jahre 1865 forderte die Gründung einer besonderen innerösterreichischen Landesgruppe und die sprachlich-kulturelle Autonomie aller in Österreich lebenden Nationen.

6 Anton Martin Slomšek (1800-1862) war der erste Bischof der neu geformten Diözese LavantMarburg, Förderer der Volksbildung, Schriftsteller und Dichter. 
dessen Errichtung sie Jahre davor noch unterstützt hatte. Der deutsche Stadtcharakter wurde auch durch die Straßenbenennung nach bekannten Deutschsprachigen und Errichtung der Denkmäler für bedeutsame deutsche Persönlichkeiten bestärkt. Infolge der Verschärfung der slowenisch-deutschen nationalen Verhältnisse in der Untersteiermark wurden deutsche Schutzvereine, Vereine zur Verteidigung und Stärkung des Deutschtums gegründet. Zu den bedeutendsten zählten der Deutsche Schulverein (1880-1918), mit der Aufgabe, das deutsche Schulwesen in der Stadt und Umgebung zu fördern, und der Verein Südmark (1889-1918), mit der Aufgabe, die wirtschaftlichen Interessen der Deutschsprachigen zu verteidigen und unterstützen.

Um die Jahrhundertwende wurde die nationale Politik in der Untersteiermark immer radikaler, die deutsch-slowenischen Beziehungen verschärften sich von Tag zu Tag. In den Jahren vor und während des Ersten Weltkriegs erreichte die antislowenische Stimmung ihren Höhenpunkt. Die Mariborer Deutschen und ihre slowenischen Unterstützer waren entscheidend gegen die slowenische Maideklaration eingestellt (1917), ${ }^{7}$ den Kaiser in Wien warnten sie vor der »hochverräterischen Politik der Slowenen« (Ferlež 2012: 56). Unmittelbar nach der Verkündung des neuen slawischen Staates der Slowenen, Kroaten und Serben (SHS, 29. Oktober 1918) beschloss der Mariborer Stadtrat, »Maribor dürfte auf keinen Fall an den slawischen Staat fallen /.../ « und erklärte, »die deutsche Stadt Marburg samt ihrer Umgebung sei aufgrund des Selbstbestimmungsrechts /.../ ein Teil der neugegründeten Republik Deutschösterreich« (ebenda: 59). Am darauffolgenden 1. November 1918 kam es in der Stadt zu einem Militärumbruch, historisch schicksalhaft wurden die Worte von Rudolf Maister, dem Offizier der altösterreichischen und später der SHS-Armee: »Maribor erkläre ich zum jugoslawischen Besitz und übernehme im Namen meiner Regierung das Militärkommando über die Stadt und in der gesamten Untersteiermark « (ebd.). Am Ende des Jahres wurde der deutsche Gemeinderat in Maribor aufgelöst und damit endete vorläufig auch die deutsche Vorherrschaft in der Stadt. Endgültig wurde Maribor wurde Teil des Königreichs der Serben, Kroaten und Slowenen ${ }^{8}$ erst mit dem Friedensvertrag von St. Germain (10. September 1919). Vorher erfolgte ein intensiver politisch-kultureller Prozess der Slowenisierung; durch Maßnahmen und Methoden, die sich von den Germanisierungsmethoden grundsätzlich nicht viel unterschieden hatten, wurden die Verwaltung sowie das Rechts- und Schulwesen in der Stadt slowenisiert. Deutsche Beamte, Lehrer, Professoren, Eisenbahner, Handwerker wurden entlassen und aus dem öffentlichen und politischen Leben verdrängt; infolge des politischen Drucks fanden die meisten Zuflucht in Österreich. Im Vergleich mit dem Jahr 1910 wiesen die Ergebnisse der Volkszählung aus dem Jahr 1921 eine Verringerung der Bevölkerung mit deutscher Umgangssprache in der gesamten Untersteiermark um das 10-fache auf. Erneut erfolgte eine statistische Assimilierung, diesmal die Assimilierung der Deutschen, unterstützt durch die manipulative Methodologie, indem nicht die vorherrschende Umgangssprache, sondern die

7 Im Mai 1917 stellten die südslawischen Abgeordneten im Wiener Reichsrat (Anton Korošec) die Forderung, alle von Slowenen, Kroaten und Serben besiedelten Gebiete innerhalb der Monarchie zu vereinigen und Selbstbestimmungsrecht zu sichern.

8 Ursprünglicher Name des Königreichs Jugoslawien (1918-1941). 
jeweilige Muttersprache ermittelt wurde. Als Deutsche gehalten wurden dementsprechend nur Bewohner mit nachweisbarer deutscher familiärer Herkunft.

Einen erneuten Aufstieg der Deutschen in Maribor und in der Untersteiermark ermöglichte der Nationalsozialismus in den 30er Jahren. Manche der Mariborer Deutschen waren Mitglieder des Schwäbisch-deutschen Kulturbundes, ${ }^{9}$ dessen Hauptaufgabe darin bestand, die deutsche Kultur im weitesten Sinne zu verbreiten und für deutsche Lehrer und Priester Sorge zu tragen. In der Tat entwickelte er sich zu einer der aktivsten, einflussreichsten und militantesten Organisationen im slowenischen Raum. Zwischen 1941 und 1945 waren viele Deutsche in Maribor und in der Untersteiermark Sympathisanten der deutschen Okkupationsmacht (Potočnik 1999), obgleich diese Feststellung nicht generalisiert werden sollte. Manche einflussreiche Mariborer Deutsche, unter anderem der Bierbrauer Tscheligi ${ }^{10}$ und evangelischer Pfarrer Baron, bei Kriegsausbruch sogar Leiter der kulturbündischen Regionalgruppe,${ }^{11}$ äußerten sich deutlich und öffentlich gegen den Terror und die Gewalt deutscher Besatzungsmacht gegen die slowenische Bevölkerung. Daran lässt sich nachvollziehen, dass die Zusammenarbeit mancher Mariborer Deutschen mit den Okkupanten eine Art pragmatische Entscheidung war, da sie sich, verglichen mit dem Status einer unerwünschten Minderheit im jugoslawischen Staat SHS, im zukünftigen Dritten Reich ein besseres Leben erhofften (Ferlež 2012: 80, Ferlež 2017).

Nach dem Zweiten Weltkrieg wurden in Maribor gebliebene Deutsche, Vertreter einer autochthonen Minderheit, und gleichermaßen prodeutsch orientierte Slowenen (Deutschtümler) systematisch vereitelt und aus dem Land vertrieben. Die Vertreibung wurde gesetzlich geregelt, ihr gesamter Besitz wurde verstaatlicht, das Staatsbürgerund Wahlrecht wurde ihnen genommen. Bis Ende 1946 liefen Deportationen nach Österreich, viele wurden verhaftet. Laut der ersten Volkszählung nach dem Zweiten Weltkrieg gab es in der Stadt und Umgebung nur noch 532 Angehörige deutscher bzw. österreichischer Nationalität (Ferlež 2012: 83). In der Öffentlichkeit sprachen sie in der Regel nicht Deutsch, zum nationalen Status äußerten sie sich aus persönlichen und politischen Gründen sehr selten. ${ }^{12}$

9 Der Schwäbisch-Deutsche Kulturbund (1920-1941) war Verein zur Förderung der deutschen Kultur und Repräsentation der Deutschen im jugoslawischen Königreich.

10 Vier Generationen der berühmten Familie Tscheligi besaßen zwischen 1841 und 1945 eine Bierbrauerei im Zentrum der Stadt.

11 Johann (Hans) Baron war Pfarrer der evangelischen Kirchgemeinde in Maribor. Als entschlossener Kämpfer um deutsche Minderheitenrechte und zeitweiliger Leiter des Schwäbisch-deutschen Kulturbundes in der Stadt war er derjenige, der 1941 den deutschen Okkupanten die Macht über die Stadt offiziell übergab.

12 Križman (2002) berichtet über Vorbehalte seiner Informanten, die sie bezüglich der Erforschung ihrer deutschen Umgangssprache (Mariborer Deutsch) hatten. Germanist an der Universität Maribor Mirko Križman (1932-2014) befasste sich eingehend mit Untersuchungen zur deutsch sprechenden autochthonen Bevölkerung an der slowenischen und der slowenisch sprechenden autochthonen Bevölkerung an der deutschen Seite der Grenze zu Österreich, vgl. Križman (1997). 


\subsection{Sprache im Schulwesen}

Die Sprache ist immer ein erstklassiges politisches Instrument. In Maribor zeigte sich diese Rolle der Sprache in der zweiten Hälfte des 19. und in der ersten Hälfte des 20. Jahrhunderts, als sie zum Zweck der Entnationalisierung und allseitiger Durchsetzung der einen oder anderen Sprachgemeinschaft instrumentalisiert wurde. So setzten sich beide Sprachen, Slowenisch und Deutsch, jeweils zu politisch günstiger Zeit, systematisch und in der Regel mit Gewalt als Lehrsprache und/oder Lehrfach in Bildungseinrichtungen aller Stufen durch.

Angesichts der wirtschaftlichen und politischen Überlegenheit der Deutschen war Slowenisch in der frühen Geschichte des Schulwesens in Maribor überwiegend in untergeordneter Stellung beteiligt. Nach einer kurzen Zeit der utraquistischen Schulen mit gemischtsprachigem Unterricht (deutsch und slowenisch) kam es Ende des 19. Jahrhunderts zur Abschaffung slowenischer Klassen und des Slowenischen als Lehrfach. 1901 verwies das katholisch-konservative Wochenblatt Slovenski gospodar ${ }^{13}$ explizit auf die Macht der Sprache in ihrer Germanisierungsfunktion: Zuerst wird Deutsch zur einzigen Lehrsprache in höheren Klassen der Volksschule, mit der Zeit geschieht dasselbe in den niedrigeren Klassen; daraufhin werden einheimische Lehrer abgesetzt und die schlimmsten Gegner als Erzieher slowenischer Kinder angestellt; zuletzt kommt es zum ständig fortschreitenden Entstellen unseres Volkes. In vergleichbarer Weise wiederholte sich die Geschichte zur Zeit des Nazismus während des Zweiten Weltkrieges, die kritische Überlegung zur Rolle der Sprache als politisches Instrument weist aber ungewollt auch auf die Parallelen mit der heutigen Zeit hin, nämlich auf ansteigende Tendenz zur englischsprachigen „Internationalisierung“ des slowenischen Hochschulwesens.

\subsection{Kulturelle Einrichtungen}

Die Sprache spielte in Maribor eine entscheidende Rolle auch bei der Gründung kultureller Vereine und Einrichtungen im 19. Jahrhundert. Zu Beginn ihres Wirkens waren national-sprachliche Unterschiede, Ab- oder Ausgrenzungen wegen der Sprache und Nationalität nicht bemerkbar, zu Ende der Donaumonarchie waren zahlreiche Vereine dagegen nationalpolitisch und somit auch sprachlich gänzlich geteilt.

Deutsch gesinnte Kulturkreise vereinten im 19. Jahrhundert bürgerliche Bewohner des Stadtzentrums innerhalb der Stadtmauer und ebenso die entstehende Arbeiterschaft, organisiert in der Grazer Sozialdemokratischen Partei und wohnhaft vor allem in der sog. Arbeiterkolonie, einer Wohnsiedlung für zahlreiche aus Österreich und Tschechien zugewanderte Arbeiter des größten damaligen Industrieunternehmens in Maribor, der Werkstätte für die Instandhaltung des Fuhrparks der Südbahnstrecke Wien-Triest. In der Zeit vor der intensiven nationalen Differenzierung in den 60er Jahren waren kulturell besonders aktiv das deutsche Theater (1785-1945), das seit 1864 unter der Schirmherrschaft des Theater- und Casinovereins wirkte und der 1846

13 Slovenski gospodar (1867-1941) war ein katholisch-konservatives Wochenblatt für ländliches Publikum in der Steiermark. Herausgegeben in Maribor nahm es eine bedeutende Rolle bei der nationalen Aufklärung an der nördlichen Grenze des slowenischsprachigen Gebiets ein. 
gegründete Männergesangverein, der zu den ältesten Gesangvereinen der Habsburger Monarchie zählte. An Theater- und Musikveranstaltungen beteiligt waren zu jener Zeit auch manche Slowenen, die sich jedoch in Einklang mit der nationalen Erwachung immer mehr in parallelen slowenischen Vereinen und Einrichtungen versammelten. Besonders hervorzuheben ist hierbei der Slawische Lesesaal (Slovanska čitalnica) (1861-1941), die zentrale kulturelle Einrichtung zur Unterstützung der Bildung und kultureller Betätigung der Slowenen sowie der 1903 gegründete Historische Verein für die Slowenische Steiermark (Zgodovinsko društvo za Slovensko Štajersko), Herausgeber der historischen Fachzeitschrift Časopis za zgodovino in narodopisje, die seit 1904 regelmäßig erscheint.

Mit der anwachsenden nationalen Differenzierung ging auch die sprachlich bedingte Verdoppelung des kulturellen Lebens in der Stadt überein. Parallel zu dem deutschen professionellen Stadttheater kam es zu den ersten slowenischsprachigen Theatervorstellungen (1865); das slowenischsprachige Laientheater erlebte einen Aufschwung mit der Gründung des Theatervereins (Dramatično društvo, 1867). Laut Stavbar (1998: 74) waren das deutsche professionelle und das slowenische Laientheater dem Status und der Qualität nach wahrscheinlich ziemlich gleichrangig, es steht aber fest, dass die Besucher des deutschen Theaters bis zu einem Drittel auch Slowenen waren.

National und sprachlich doppelt geprägt waren in der zweiten Hälfte des 19. Jahrhunderts auch Zeitungen. Mit der Geschichte der Deutschen in Maribor und in der gesamten Untersteiermark aufs Engste verbunden ist die deutsche Marburger Zeitung (1862-1945), slowenisch auch Marburgerica (,Marburgerin') genannt, die bestimmt auch von Slowenen gelesen wurde. Unter slowenischen Zeitungen seien erwähnt das bürgerlich orientierte Blatt Straža (,Wache') (1909-1927) sowie die Zeitung Slovenski gospodar (,Slowenischer Herr') mit dem Untertitel Podučitveni list za slovensko ljudstvo (,Belehrendes Blatt für slowenisches Volk ${ }^{6}$ ), erschienen zwischen 1867 und 1941 und vor allem der untersteirischen ländlichen Bevölkerung gewidmet. In der Marburger Zeitung findet man einige seltene gedruckte Texte in Mariborer Deutsch.

\section{SPRACHEN IM »DEUTSCHEN« MARIBOR}

\subsection{Das Mariborer Deutsch}

Der permanente und dauerhafte Sprachkontakt zwischen den dialektalen und standardsprachlichen Varietäten des Slowenischen und Deutschen sowie die intensive Zuwanderung aus den deutschsprachigen Gebieten und dem slowenischsprachigen Umland führten in der Stadt zu starken gegenseitigen Beeinflussungen zwischen den beiden Sprachen. Überreste dieser sprachlichen Wechselwirkungen sind in der Mariborer Umgangssprache leicht nachvollziehbar. Im weiteren Verlauf des Beitrags wird eine spezifische dialektale Ausprägung der deutschen Umgangssprache skizziert, die in der Zeit der Habsburger Monarchie, allerdings auch später in Maribor gesprochen wurde und üblicherweise unter dem Namen Marburger Deutsch bekannt ist. Die Zahl der Sprecher ging nach dem Ersten und vor allem nach dem Zweiten Weltkrieg stark zurück, sodass es heute voraussichtlich keine Sprecher des Mariborer Deutsch mehr gibt. 
Spricht man über das Mariborer Deutsch, so meint man damit eine überwiegend gesprochene und durch den dialektalen Einfluss zahlreicher deutschsprachiger $\mathrm{Zu}-$ wanderer geprägte Varietät des Deutschen. Die Zuwanderung aus der österreichischen Steiermark und anderen österreichischen Ländern brachte stark beeinflussende Wirkung auf die städtische deutsche Umgangssprache mit sich. Das Mariborer Deutsch interpretiert man als eine südbairische Mundart mit archaischen deutschen Besonderheiten und deutsch-slowenischen Interferenzerscheinungen (Križman 2002). Scherzhaft, allerdings auch pejorativ wurde es als Kucheldeutsch gekennzeichnet, verwendet für den privaten Umgang im kleinbürgerlichen Milieu; dazu wurde es bemerkenswert geprägt von den slowenischen Mundarten der Mariborer Umgebung. Die Sprecher differenzierten es deutlich von der deutschen Standardsprache, die in den Schulen und Zeitungen vertreten war. ${ }^{14}$ Sprachkontaktbedingt waren einige Interferenzerscheinungen, darunter der Einfluss der slowenischen Syntax, vgl. die Wortfolge in (1), deutlich beeinflusst durch die syntaktische Regelung des Slowenischen oder die Einwirkung der slowenischen Morphologie, vgl. die Übernahme des slowenischen grammatischen Genus beim deutschen Nomen in (2):

(1) Wir habn gewohnt in Brunndorf, in anem oltn Haus; standardsprachlich: Wir haben in Brunndorf gewohnt, in einem alten Haus; slowenisch: Živeli smo na Studencih, $v$ stari hiši; muos $i$ hölfn; standardsprachlich: ich muß helfen; slowenisch: moram pomagati;

(2) die Spotn/der Spotn; standardsprachlich: der Spaten; slowenisch: lopata (weiblich); die Dom; standardsprachlich: der Dom; slowenisch: stolnica (weiblich), vielleicht auch nach die Domkirche, slowenisch: stolna cerkev (weiblich).

Phonetische Eigenheiten bairischer Dialekte zeigen sich als Verlust der Stimmhaftigkeit bei stimmhaften Verschlusslauten im Anlaut, vgl. Poch : Bach, Puach : Buch, Picha/Piacha: Bücher, Paumwolle : Baumwolle; als Diphtongisierung, vgl. Muata: Mutter, Gauns : Gans, guat : gut, Pluat : Blut, reidn : reden, Tuo mir den Knoupf aunnähn : Tu mir den Knopf annähen; als Lautwandel von a zu o, vgl. Poch : Bach, Weingortn: Weingarten, Schof: Schaf, Wolt : Wald, Nosn : Nase, hob : habe; als Ersatz des wortauslautenden -er durch - $a$, vgl. dea Pfora : der Pfarrer, Vota : Vater, unta : unter.

Auf bairische morphologische Merkmale hinzuführen sind Generalisierung der Basen bei der starken und schwachen Deklination, und zwar durch die Reduktion des unbetonten wortauslautenden Vokals bzw. durch die Generalisierung des $n$-Suffixes

14 Eine vergleichbare Situation beobachtet man in mehreren Städten im Südosten Europas, dazu zählt z. B. die gesprochensprachliche dialektale Mischung des Deutschen und des Tschechischen in Wien und Prag (Deutsch, geprägt durch tschechische syntaktisch-lexikalische Elemente, auch Kucheldeutsch genannt und in Prag des 19. Jahrhunderts gesprochen sowie die Wiener Variante der deutsch-tschechischen Mischung, bekannt unter dem Namen Bömackeln, vgl. Hanisch 2015); unter Anderem ist diese Sprachmischung in der deutschen Übersetzung Des braven Soldat Schwejk erhalten. Vgl. auch das Kucheldeutsch in der Stadt Sibiu (Hermannstadt) im rumänischen Siebenbürgen und Umgangssprachen deutscher Bevölkerung in anderen Städten Osteuropas (Osijek, Požarevac und mehrere Städte im heute serbischen Banat). Ausführlich dazu Gehl (1997), der das Mariborer Deutsch allerdings nicht erwähnt. 
(Braune 1987: 192, 204), vgl. Stroßn : Straße, Wiesn : Wiese, Kirchn : Kirche, Kotz : Katze; auch durch eine disproportionale Häufung der Diminutiva auf -(er)l, vgl. Winzerl : Winzer, Hoamatl : Heimat, Wirst a Eierl essn? : Wirst du ein Ei essen? und durch die Reduktion des Halblautes $e$ im Präfix ge- bei Perfektpartizipien, vgl. gwußt : gewußt, gaungen : gegangen, gsprochen : gesprochen, kobt: gehabt.

Autochthone Sprecher des Mariborer Deutsch, die in der empirischen Untersuchung Križmans mitgewirkt haben (Križman 2002: 161), waren sich der Unterschiede zu der deutschen Standardsprache, die sie in der Schule lernten, bewusst; diese empfanden sie als „,schönes Sprechen“: „Man kennt jedn Untaschteira, wenn ea den Mund aufmacht. I hob mir eigentlich nie bemüht schen zu reidn, hob i ima dos Untaschteirische mitkschleppt.“ (ebd.) Zugleich betonten sie die Tatsache, dass das Mariborer Deutsch die Umgangssprache ihres privaten Umfeldes in der Kindheit war: „I hob daitsch gsprochen. Mein Vota hot keine Ahnung kobt von der slowenischen Sproch.“ (ebd.)

Geschrieben (und gedruckt) wurde das Mariborer Deutsch selten; bekannt ist die dialektale deutsche Lyrik, veröffentlicht in der damaligen deutschen Presse (Marburger Zeitung, Marburger Adreßkalender, Deutscher Bote). Zum Teil sind es Kinderlieder, andererseits überwiegen engagierte, emotional-expressive, pathetische Gedichte aus der Zeit des Ersten Weltkrieges (unter ihnen Gedichte der in Celje geborenen Autorin Margareta Glantschnigg, geh. Weinhandl, 1880-1975). Ihre sprachlich-dialektale Dimension kann unter Berücksichtigung des damaligen Zeit-Raumes als Ausdruck des deutschen Patriotismus und der Liebe zur deutschen Sprache interpretiert werden (vgl. Križman 2002: 166).

\subsection{Das ,deutsche“ Slowenisch}

Überreste des permanenten und intensiven deutsch-slowenischen Sprachkontakts findet man zahlreich auch in der substandardsprachlichen Umgangssprache der Stadt Maribor. ${ }^{15}$ Unübersehbar sind viele Germanismen; in Anbetracht der wirtschaftlichen Entwicklung der Stadt gehört dazu vor allem die Lexik, gebunden an Handwerk, Küche, Kleidung und Haushalt, weniger vertreten sind dagegen Ausdrücke, gebunden an Tiere, Pflanzen, Ackerbau (Zorko 2002, 143), obgleich deutsche Einflüsse auch hier nachvollziehbar sind. Deutscher Herkunft sind dementsprechend:

(1) Benennungen, gebunden an Menschen und das alltägliche Leben, vgl. ksiht ${ }^{16}$ (,Gesicht'), pukl (,Buckel'); flanca (,Pflanze'), murka (,Gurke'); puter (,Butter'), cuker (,Zucker'), šnops (,Schnaps'); župa (,Suppe'), zos (,Soße'), knedl (,Knö-

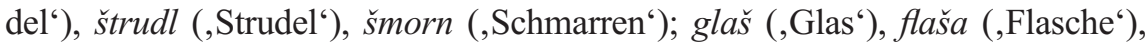
šalca (,Schalle'), beštek (,Besteck'); štumfi (,Strümpfe'), zokni (,Socken');

15 Viele von den im weiteren Textverlauf genannten Ausdrücke sind nicht nur für die steirischen Mundarten charakteristisch, denn gleichermaßen finden sie sich in manchen Dialekten des slowenischen Sprachraumes, vgl. dazu Striedter-Temps (1963), Šekli (2015).

16 Wortbeispiele sind lediglich illustrativ. 
(2) Lexik, gebunden an Handwerk und Wirtschaft, ${ }^{17}$ auch Berufsbenennungen, vgl. bremza (,Bremse'), blinker (,Blinker'), štender (,Ständer'), dekl (,De-

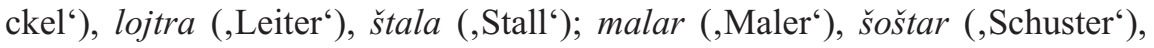
tišler (,Tischler');

(3) Verben mit entsprechenden Nominalisierungen, vgl. ajnkaufati/ajnkauf (,einkaufen/Einkauf'), luftati/luft (,lüften/Luft'), špilati/spil (,spielen/Spiel');

(4) Lexik zum Ausdruck von Eigenschaften, Merkmalen, vgl. ajnfoh (,einfach'), fertik (,fertig'), na knap (,knapp'), bek (,weg').

In syntaktischer Hinsicht ist der Einfluss des Deutschen vor allem in der Form lehnübersetzter adverbialer Verbverbindungen auffallend, vgl. gor fstat (aufstehen), dol past (hinunter-, nach unten fallen) und verbaler Phrasen nach dem deutschen Muster $z u$ + Verb, vgl. to je za gor postavit, to ni za govorit.

Die historisch-etymologische Betrachtung der Germanismen in der dialektal geprägten Umgangssprache der Stadt Maribor wirft die Frage auf, inwiefern sie auch in der Gegenwart verwendet werden. Anhand neuerer Forschungsdaten (Lipavic Oštir 2014, 2017) wird deutlich, dass sie als hochgradig verständlich gehalten werden (die Befragten kannten im Durchschnitt mehr als $90 \%$ der Lexik ,deutscher" Herkunft), dass sie aber seltener Verwendung finden (im Durchschnitt um $65 \%$ ), dies in der Regel innerhalb der gesprochenen substandardsprachlichen Varietäten. Das Alter der Befragten spiele hierbei keine wesentliche Rolle, was für eine generationsunabhängige Gefestigtheit des sprachlich-kulturellen Erbes sprechen könnte. Trotz alledem führt die Stadt Maribor mit der deutschen Sprache an sich und mit dem Mariborer Deutsch noch insbesondere eine seltsame, eigenartige Beziehung. In Toti list, dem Mariborer humoristischen Blatt, wird sie etwa eingesetzt, um ironische, satirische, potentiell humorige Effekte zu erzielen, offensichtlich geht es aber auch um den Ausdruck spöttischer, leicht verachtender Haltung gegenüber ihren autochthonen Sprechern in Maribor. Vgl. hierzu einen Textausschnitt aus dem Jahr 1953: ... da rajn nič več od nas not v cajtunge ne nadrukajo. Za vsakršne sorte folk majo plac, samo za nas, kuhltajčare, ni placa v cajtungah! Saj smo mi glih tak, če se ži tak vzame, ene sorte nacional mindervertigkeit, göl? Te smo se že unlenkst menli od tega v naši gasi, da bi se zrihtali mi sami, pa alajnik ene cajtunge začeli drukat, iberhaupt sploh nur v kuheltajč! Toti že tak ma furtafurt kaj za nerglat kaj je noviga, no kaj vse naprej pride v Marpurki. Kera se cumbajšpil s kerim ženi, kera bo s kerim mela pankerta, kera keriga s kerim petriga ...

\section{ZUM SCHLUSS}

»Beide Weltkriege wie auch die Nachkriegsjahre sind das tragische Ergebnis der überbewerteten Nationalgefühle, die verhängnisvoll in das Leben der Mariborer eingegriffen und eine wesentliche Auswirkung auf Maribor hatten«, so Ferlež in ihrer Überlegung zur schicksalhaften Geschichte der Deutschen samt ihrer Sprache und Kultur im

17 Da die wachsende Industrie in der Stadt gänzlich in deutschen Händen war, war auch der gesamte technische Wortschatz durch Germanismen durchdrungen. 
historischen Maribor (Ferlež 2012: 15). Autochthone Sprecher des Mariborer Deutsch gibt es nicht mehr, dazu ist auch das Forschungsinteresse für die deutsche Stadtbevölkerung und ihr durchaus reiches und vielfältiges Kulturerbe relativ bescheiden. Die Thematik scheint erst in den letzten Jahren intensiver untersucht zu werden; genannt seien partielle historische, ethnologische und soziolinguistische Untersuchungen sowie Studien zum Deutschen Theater (Bruno Hartman, Matjaž Birk) bzw. zum Musikleben (Manica Špendal) innerhalb deutscher Vereine und Einrichtungen im 19. Jahrhundert. Eine ganzheitliche, interdisziplinäre und vor allem ideologisch unbelastete Auseinandersetzung mit dem deutschen sprachlichen und kulturellen Erbe in der Stadt und Region steht allerdings noch aus. Pungartnik (2016) stellt empört-resigniert fest, wie wenig man deutsch schreibende Literaten der Region kennt, wie wenig man über Gelehrte, Intellektuelle und Künstler deutscher Herkunft weiß, obgleich sie in ihrem Wirken mit dem jahrhundertelang deutschen Maribor bzw. der Untersteiermark verbunden waren und das heutige slowenische Land kulturell bereichert und folgenreich geprägt haben. Die bemerkenswerte Ausstellung mit dem Titel Deutsche und Maribor (Ferlež 2012; Maribor, Europäische Kulturhauptstadt 2012) war ein Schritt in die richtige Richtung.

\section{Literatur}

BRAUNE, Wilhelm (1987) Althochdeutsche Grammatik. Bearbeitet von Hans Eggers. Tübingen: Max Niemeyer Verlag.

BUNC, Stanko (1963) „O nastanku, razvoju in pomenu priimkov.“ Jezik in slovstvo, $8 / 6,174-177$.

CURK, Jože (2002) „O prebivalstvu Maribora med sredinama 18. in 19. stoletja.“ Maribor skozi čas I. = Studia Historica Slovenica 2/1, 43-78.

CVIRN, Janez (2016) »Das Festungsdreieck«. Zur politischen Orientierung der Deutschen in der Untersteiermark. Wien: LIT Verlag.

FERLEŽ, Jerneja (2002) „Prebivalstvo Maribora 1848-1991.“ Maribor skozi čas I. = Studia Historica Slovenica 2/1, 79-125.

FERLEŽ, Jerneja (Hrsg.) (2012) Deutsche und Maribor. Ein Jahrhundert der Wenden: 1846-1946. 1. Ausg. Maribor: Umetniški kabinet Primož Premzl.

FERLEŽ, Jerneja (2017) „Spremembe konteksta, premene vlog: Nemci in Slovenci v Mariboru od srede 19. do srede 20. stoletja.“ In: S. Poljak Istenič/M. Habinc/K. Hrobat Virloget (Hrsg.), Nemi spomini: manjšine med obrobjem in ospredjem. Ljubljana: Slovensko etnološko društvo, 72-86.

GEHL, Hans (1997) Deutsche Stadtsprachen in Provinzstädten Südosteuropas. Stuttgart: Franz Steiner Verlag.

HANISCH, Klaus (2015) „Das Deutsch der Tschechen. Mischsprachen beschäftig(t)en Zuwanderer, Literaten - und gerade auch Sprachwissenschaftler." Prager Zeitung (12.03.2015). https://www.pragerzeitung.cz/das-deutsch-der-tschechen/

JENUŠ, Gregor (2011) Ko je Maribor postal slovenski: Iz zgodovine nemško-slovenskih odnosov v Mariboru od konca 19. stoletja in v prevratni dobi. Maribor: Znanstvenoraziskovalni inštitut dr. Franca Kovačiča. 
JESENŠEK, Vida (2019) „Nemščina v Mariboru: preplet kulturnozgodovinskih in jezikovnih danosti.“ In: M. Šekli, L. Rezoničnik (Hrsg.), Slovenski jezik in njegovi sosedje. Ljubljana: Zveza društev Slavistično društvo Slovenije, 178-185.

KRIŽMAN, Mirko (1997) Jezikovna razmerja: Jezik pragmatike in estetike v obmejnih predelih ob Muri. Maribor: Slavistično društvo.

KRIŽMAN, Mirko (2002) „Mariborska nemščina.“ Maribor skozi čas I. = Studia Historica Slovenica 2/1, 151-178.

LIPAVIC OŠTIR, Alja (2014) „Germanizmi v vsakdanji rabi slovenskega jezika.“ In: M. Jesenšek (Hrsg.), Jeziki, literature in kulture v stiku. Maribor: Univerza, 209228.

LIPAVIC OŠTIR, Alja (2017) Substandardsprachliche Germanismen im Slowenischen. Wien: LIT.

POTOČNIK, Dragan (1999) „Mariborski Nemci v letih 1918-1941.” Kronika 1-2, $143-151$.

PUFF, Rudolf Gustav (1847) Marburg in Steiermark, seine Umgebung, Bewohner und Geschichte. Gratz: Andr. Leykam'schen Erben.

PUNGARTNIK, Marjan (2016) „Drugi obraz Maribora.“ Locutio 94 (01.11.2016). https://www.locutio.si/index.php?no=94\&clanek $=2900$

STRIEDTER-TEMPS, Hildegard (1963) Deutsche Lehnwörter im Slowenischen, Wiesbaden: Harrassowitz.

STAVBAR, Vlasta (1998) Kulturno dogajanje v Mariboru v letih 1914-1918. Maribor: Založba Obzorja.

ŠEKLI, Matej (2015) „Pomenska polja nemških izposojenk v slovenščini.“ Jezikoslovni zapiski 21, 2-31.

ZORKO, Zinka (2002) „Mariborski pogovorni jezik.“ Maribor skozi čas I. = Studia Historica Slovenica 2/1, 127-150.

\section{Zusammenfassung \\ DEUTSCH IM HISTORISCHEN MARIBOR}

Der jahrhundertelange Kontakt zwischen der slowenischen und deutschen Sprache und Kultur hat die Stadt Maribor bedeutsam geprägt. Seit den Anfängen der Stadtentwicklung im 12. Jh., vor allem aber seit dem 13. Jh., als die Stadt unter die Herrschaft der Habsburger kam, nahm die Zuwanderung aus den deutschsprachigen Ländern stark zu, vorrangig aus dem heutigen österreichischen Kärnten und der österreichischen Steiermark, jedoch auch aus Bayern und vielen deutschen mittelalterlichen Städten, was zu einem stark ausgeprägten deutschen Stadtcharakter führte. Die deutschsprachigen $\mathrm{Zu}$ wanderer waren in der Regel hochqualifiziert und gebildet; sehr schnell übernahmen sie führende Positionen in der Wirtschaft und im öffentlichen Leben. Zu bemerkbaren national- und kulturpolitischen Auseinandersetzungen zwischen den Deutschsprachigen und den Slowenen kam es in den 60er Jahren des 19. Jh. Seitdem dienten beide Sprachen, jeweils zu ihrer Zeit, zur Identifizierung der Stadtbewohner mit eigener 
Sprach- und Nationalgemeinschaft sowie zur Regelung sozialer und Machverhältnisse in Wirtschaft, Verwaltung, Alltagskommunikation, Schulwesen und Kulturleben. Die gegenseitige Beeinflussung beider Sprachen führte zur Ausprägung einer umgangssprachlichen Varietät des Deutschen mit slowenischen Interferenzerscheinungen, bekannt unter dem Namen Mariborer Deutsch sowie zur Ausbildung der städtischen slowenischen Umgangssprache mit zahlreichen Germanismen.

Schlüsselwörter: Deutsch, Maribor, Mariborer Deutsch, slowenische Umgangssprache, Germanismen

\section{Abstract \\ GERMAN IN THE HISTORY OF MARIBOR}

Since the beginning of the development of the city of Maribor in in $12^{\text {th }}$ century, the contacts between the Slovene and German languages and cultures have had a great impact on its history. This German cultural influence became particularly evident in $13^{\text {th }}$ century when the city came under the rule of the Habsburgs and, as a consequence, received numerous immigrants from German-speaking countries, primarily from what are today the Austrian land of Carinthia and Styria, but also from Bavaria and several German medieval cities. This contributed to the shaping of the heavily Germanized character of the city. The German-speaking newcomers were usually highly qualified and quickly took on leading positions in business and public life. In the 1860s, Maribor witnessed some violent national, political and cultural conflicts between its Germanspeaking and Slovene-speaking citizens. Since then, both languages have served as markers of identity and as means of political expression for its citizens in everyday communication as well as in educational and cultural settings. The intense contact between the two languages gave rise to a colloquial variety of German with Slovene interference (Maribor German) and to a variety of colloquial Slovene characterized by numerous Germanisms.

Keywords: German, Maribor, Maribor German, colloquial Slovene language in Maribor

\section{Povzetek NEMŠČINA V ZGODOVINSKEM MARIBORU}

Maribor je zaznamovan s stikom dveh jezikov in dveh kultur, slovenske in nemške. Že od začetkov njegovega razvoja v 12. st., predvsem pa od 13. st., ko je pristal pod oblastjo Habsburžanov, viri izpričujejo številna priseljevanja iz nemško govorečih dežel, predvsem iz današnje avstrijske Koroške in Štajerske, Bavarske, pa tudi iz drugih srednjeveških nemških mest, kar je mestu dajalo močan nemški značaj. Večinsko nemško 
govoreči priseljenci so bili praviloma kvalificirani in izobraženi; hitro so dobili v roke gospodarstvo in javno življenje v mestu. Do ostrejšega narodno-političnega razlikovanja med nemško in slovensko govorečimi prebivalci Maribora je prišlo v 60-ih letih 19. st. Od takrat sta oba jezika, vsak v svojem času, služila opredeljevanju narodnostne pripadnosti, z njuno pomočjo se je merila moč v vsakdanjem sporazumevanju, šolstvu in kulturi, izrabljana sta bila kot prvovrstno sredstvo političnega boja. Intenzivni medjezikovni stiki so pripeljali do oblikovanja pogovorne mariborske različice nemščine s prepoznavnimi slovenskimi interferenčnimi vplivi in hkrati $\mathrm{k}$ formiranju pogovorne, $\mathrm{s}$ številnimi germanizmi prepletene mariborščine.

Ključne besede: nemščina, Maribor, mariborska nemščina, mariborski pogovorni jezik, germanizmi 